\title{
Puntos críticos de la cadena productiva de la mora (Rubus glaucus Benth), en el municipio de Pamplona, Colombia
}

\section{Critical points of change in the blackberry productive chain (Rubus glaucus Benth) in the municipality of Pamplona, Colombia}

Yolanda González-Castro'
Omaira Manzano-Durán
Obdulio García-Hoya $^{2}$

Recibido: noviembre 30 de 2018

Aceptado: junio 28 de 2019

\begin{abstract}
Resumen
Se realizó un diagnóstico a la cadena productiva de la mora (Rubus glaucus Benth), en el municipio de Pamplona, Colombia, mediante un enfoque mixto. En lo cuantitativo, se estudiaron las variables: investigación, buenas prácticas agrícolas y manejo de pos cosecha. En lo cualitativo, se estudiaron las debilidades de la cadena productiva y mejoras que se requieren, a partir de entrevistas a expertos. Como resultado se obtuvo que la cadena productiva de la mora presenta fortalezas, como: suficiencia de espacios para la siembra, adaptación y rendimiento del fruto a los suelos, consideraciones del valor medicinal y nutricional del producto, entre otras. Sin embargo, se encuentran puntos críticos en cuanto a: la mejora genética de los cultivos, faltan rutinas para el análisis de agua y desinfección adecuada, junto con malas prácticas en el manejo técnico del cultivo. Igualmente, se evidencia que los productores no utilizan herramientas administrativas ni llevan registros contables que orienten la toma de decisiones.
\end{abstract}

Palabras clave: agricultura, cadena productiva, investigación agrícola, cultivo, mora.

\begin{abstract}
A diagnosis of the blackberry (Rubus glaucus Benth) production chain was made in the municipality of Pamplona, Colombia, using a mixed approach. In quantitative terms, the following variables were studied: research, good agricultural practices and post-harvest management. Qualitatively, the weaknesses of the production chain and the improvements required were studied, based on interviews with experts. As a result, it was obtained that the blackberry productive chain presents strengths, such as: sufficiency of spaces for sowing, adaptation and yield of the fruit to the soils, considerations of the medicinal and nutritional value of the product, among others. However, there are critical points regarding to the genetic improvement of crops, lack of routines for water analysis and adequate disinfection, along with bad practices in the technical management of the crop. It is also evident that producers do not use administrative tools or keep accounting records to guide decision-making.
\end{abstract}

Keywords: agriculture, production chain, agricultural research, cultivation, blackberry.

1 Administradora de Empresas, Doctora en Administración de Negocios, Universidad Nacional Abierta y a Distancia, Pamplona, Colombia. E-mail: yolanda.gonzalez@unad.edu.co ORCID: 0000-0002-9497-7132

2 Licenciada en Matemáticas y Física, Doctora en Administración de Negocios, Universidad Nacional Abierta y a Distancia, Ocaña, Colombia. E-mail: omaira.manzano@unad.edu.co ORCID: 0000-0002-2715-8903

3 Administrador de Empresas, Magíster en Administración de Organizaciones, Universidad Nacional Abierta y a Distancia, Pamplona, Colombia. E-mail: obdulio.garcia@unad.edu.co ORCID: 0000-0003-2832-9169 


\section{Introducción}

La mora (Rubus glaucus Benth) es una fruta que pertenece a las rosáceas. Se caracteriza por: ser perenne, arbustiva, de tallo rastrero o semierguidos. El fruto es un agregado de drupas, cada uno con semilla en su interior. La planta presenta floración y fructificación permanente y se adapta a alturas que van de 1200 a $3500 \mathrm{~m}$, con temperaturas de $16^{\circ} \mathrm{C} \mathrm{A} 18^{\circ} \mathrm{C}$, humedad del ambiente de 70 a $80 \%$ y precipitación de 1.200 a $1.700 \mathrm{~mm}$ anuales (Sánchez-Morales, Villares-Jibaja, Niño-Ruiz \& Ruilova, 2018). Las propiedades nutricionales de la mora son: su bajo valor calórico, rica en vitaminas C, fibra, hierro y calicio, al igual que en taninos, ácidos orgánicos y antocianos (Ronald et al, 2004, citado por Usca, 2011).

La mora se cultiva en muchas partes del mundo, y en América los países más representativos, son: Colombia, Ecuador, Estados Unidos, Guatemala, Honduras, México, Panamá y Salvador (Dotor-Robayo et al., 2016). Aunque la planta Rubus glaucus Benth, popularmente conocida como mora de castilla, es la más cultivada en Colombia, por su óptimo desarrollo y menor exigencia de mantenimiento, se han desarrollado otras variedades de la mora en otros países. Por ejemplo, en Costa Rica, se siembra la mora vino o mora uva (vino Rubus sp) con espinas y sin espinas, que es una mora criolla, adicionalmente se cultiva un tipo de mora híbrida denominada Brazos y sus características la hacen superior a la criolla (Ministerio de agricultura y ganadería de Costa Rica, 2014). Asimismo, en Ecuador se creó una nueva variedad de mora, Iniap Andimora, caracterizada por no tener espinas, facilidad para la recolección, más dulce y es superior en cuanto a las ventajas de almacenamiento (Llerena, Samaniego, Ramos \& Brito, 2014).

La cadena productiva de la mora conlleva las etapas de: preparación del material vegetal, preparación del terreno, siembra, manejo y sostenimiento del cultivo, cosecha y comercialización, y agroindustria (Miranda-Lasprilla, 2011).
En Colombia, la producción de frutas registra una de las más altas tasas de crecimiento productivo en los últimos años. Aquellas frutas como: mora, guayaba y lulo, que están destinadas a la producción de jugos, alcanzan un $12 \%$ del total de la producción nacional, según la Corporación Colombiana de Investigación Agropecuaria, Corpoica (2014). Las causas del aumento de la productividad de las frutas colombianas, está en factores como: la presencia de nichos apropiados para los cultivos, el valor nutricional y medicinal de los productos, así como también en la calidad de los suelos donde se cultivan (Miranda-Lasprilla, 2011).

El cultivo de la mora en Colombia, se realiza en forma artesanal por pequeños y medianos agricultores (Grijalba-Rativa, Calderón-Medellín \& Pérez-Trujillo, 2010). En 2011, alcanzó una extensión de $11673 \mathrm{Ha}$, con una producción de 94.303 Tn al año. Se espera para el año 2026, un incremento equivalente a 20631 ha, considerándose un cultivo promisorio para la exportación (Ayala, Valenzuela \& Bohorquéz, 2013). Los departamentos donde se registran los mayores cultivos de mora, son: Cundinamarca, Santander, Valle, Caldas, Antioquia, Huila, Tolima, Boyacá, Nariño y Norte de Santander.

En Norte de Santander (NS), departamento donde se ubica el municipio de Pamplona, en 2008, se reportaban $5234 \mathrm{Ha}$ de frutales, que generaban $9 \mathrm{Tn} / \mathrm{Ha}$ de mora, cifras que están muy por debajo del promedio nacional (Asohofrucol, 2008). En Pamplona Existe una asociación que agrupa a los principales cultivadores, denominada ASPRI, constituida por 37 productores de mora. En vista de la importancia de este cultivo para el municipio, se hace necesario identificar los obstáculos en el proceso productivo, para establecer cambios integrales que fortalezcan la cadena productiva de la mora. En este sentido, se realizó un diagnóstico participativo con el propósito de identificar aquellos factores de cambio que requiere la cadena, en aspectos como: la investigación agrícola, las buenas prácticas y el manejo de poscosecha. 


\section{Materiales y métodos}

\subsection{Ubicación del proyecto}

El diagnóstico rural participativo, se realizó en la zona rural del municipio de Pamplona, Colombia. Pamplona está ubicada en el suroccidente del departamento de Norte de Santander, se encuentra a una distancia de $75 \mathrm{~km}$ de la capital del departamento, y posee una superficie de $318 \mathrm{~km} 2$, equivalentes al $1,4 \%$ del total departamental. El clima oscila entre 12,4 y $16,9^{\circ} \mathrm{C}$. Además, se presentan dos períodos: uno de mayor temperatura que comprende los meses de marzo a noviembre, y otro de menor temperatura, entre diciembre y febrero (Gobernación Norte de Santander, 2016).

\subsection{Diseño metodológico}

El trabajo se adelantó bajo un enfoque mixto de tipo descriptivo (Zuluaga-Duque, 2017), donde se analizan tres variables: Investigación agrícola, Buenas Prácticas Agrícolas (BPA), y manejo de poscosecha. El proyecto se desarrolló teniendo en cuenta las siguientes fases:

i) Revisión documental y construcción del marco referencial.

ii) Diseño de formatos para la recolección de información y revisión de expertos. De acuerdo a las variables seleccionadas, se escogieron los instrumentos requeridos para la recolección de información. Estos documentos se validaron por dos expertos en investigación agropecuaria, del Ministerio de Agricultura y de la Universidad de Pamplona. iii) Aplicación de instrumentos. Se encuestaron 20 productores, integrantes de la Asociación de Productores, Transformadores y Comercializadores Rurales Integrados (Aspri), del municipio de Pamplona. Además, se aplicó una entrevista a 2 expertos del Instituto Colombiano Agropecuario (ICA), y de la Secretaría de Desarrollo del municipio, y se visitó el área sembrada para corroborar los resultados.

iv) Análisis de información. Se empleó el software SPSS para el análisis estadístico de fecuencias, de las 3 variables cuantitativas. Además, se usó el software Atlas Ti, para la revisión cualitativa de las entrevistas. Los resultados encontrados permitieron evidenciar las fortalezas al igual que los puntos críticos de la cadena productiva de la mora.

\section{Resultados y discusión}

\subsection{Encuestas y entrevistas}

En cuanto a la investigación agrícola, que hace referencia a: la variedad cultivada, mejora genética, valor nutricional y valor medicinal, se evidenció que la mayoría de los agricultores (45\%), concentra su producción en la variedad mora de Castilla, por su fácil adaptación y alto rendimiento. Otros cultivadores se han concentrado en la nueva variedad mora uva (10\%). Asimismo, el $40 \%$ de los productores encuestados, considera que las mejoras genéticas pueden redundar en mayor productividad, y que el producto es rico en valor nutricional y medicinal, ver tabla 1. 
Tabla 1. Variable investigación agrícola.

\begin{tabular}{|c|c|c|c|}
\hline Investigación Agrícola & & frecuencia & $\%$ \\
\hline \multirow{5}{*}{ Variedad } & Castilla & 9 & 45 \\
\hline & Castilla y mora uva & 6 & 30 \\
\hline & Criolla & 1 & 5 \\
\hline & Mora uva & 2 & 10 \\
\hline & Morón & 2 & 10 \\
\hline \multirow{2}{*}{ Mejora Genética } & $\mathrm{Si}$ & 11 & 55 \\
\hline & No & 9 & 45 \\
\hline \multirow{2}{*}{ Beneficios } & Mayor productividad & 8 & 40 \\
\hline & Sin beneficios & 12 & 60 \\
\hline \multirow{4}{*}{ Valor Nutricional } & Vitaminas & 5 & 25 \\
\hline & Rehidratante & 1 & 5 \\
\hline & Minerales & 1 & 5 \\
\hline & $N / S \circ N / R$ & 13 & 65 \\
\hline \multirow{4}{*}{ Valor Medicinal } & Aumento glóbulos rojos & 7 & 35 \\
\hline & Remedio para la gripa & 10 & 50 \\
\hline & Otros & 1 & 5 \\
\hline & $N / S \circ N / R$ & 2 & 10 \\
\hline
\end{tabular}

En este sentido, Kessel-Domini (2012), afirma que el mejoramiento genético de los cultivos permite obtener variedades más resistentes al clima y a las enfermedades, al igual que mejora en el color y sabor y valores nutricionales, lo que redunda en un producto de calidad. Estas variedades mejoradas tienen mayor probabilidad de ser comercializadas para su utilización en la industria de alimentos, ya sean estos frescos o procesados (Trujillo-Navarro, 2018).

Las Buenas Prácticas Agrícolas, BPA, se establecieron a partir de lo recomendado por el Instituto Co- lombiano Agropecuario, ICA (2016). En este caso, se constataron indicadores medios y altos en: los cultivadores cuentan con espacios adecuados para la siembra y recolección; el análisis del suelo se hace con asesoría de un técnico agropecuario; cuentan con quebradas donde pueden tomar el agua muy cerca del lugar donde siembran. Igualmente, realizan control de maleza ya sea con azadón o guadaña. Además, realizan la práctica de control de insectos y de hongos, y, aunque aplican abonos orgánicos, estos no son abonos certificados. Estas prácticas, en su conjunto, permiten mantener un producto de calidad, ver figura 1. 


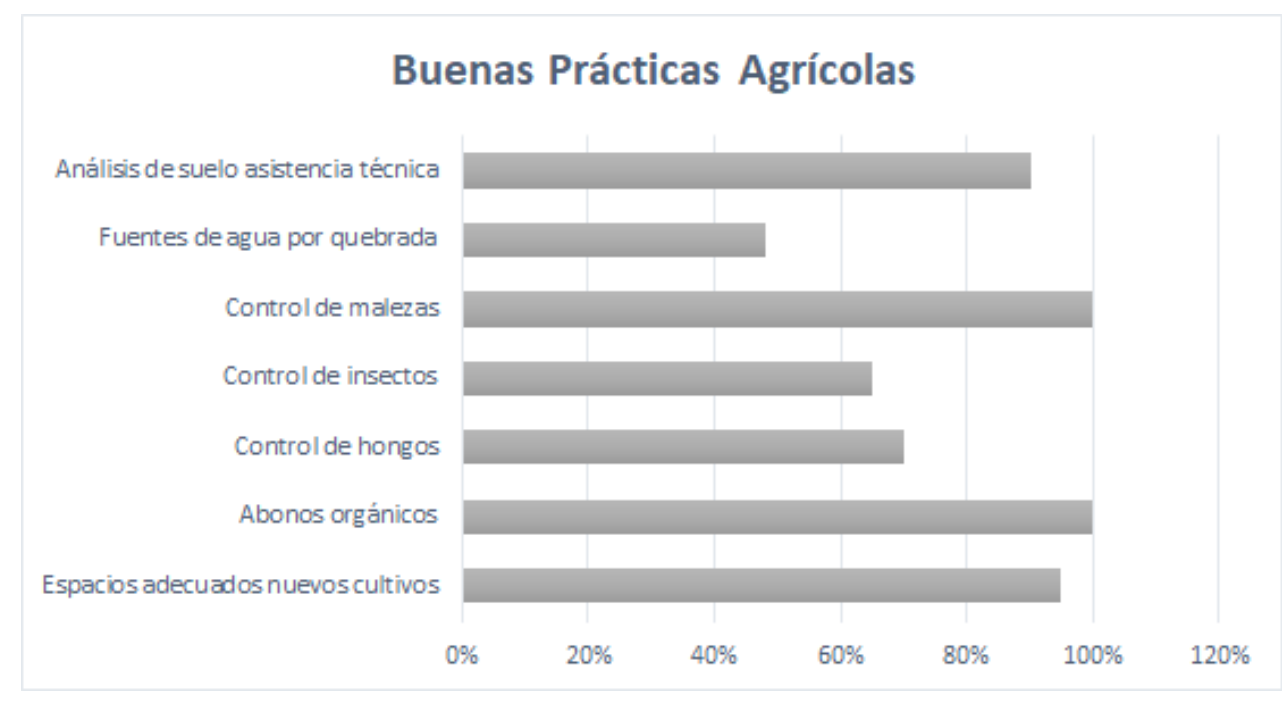

Figura 1. Buenas prácticas agrícolas en la cadena productiva de la mora (Rubus glaucus Benth).

En la tabla 2 se presentan los indicadores con bajo valor, y por tanto se consideran puntos críticos en la cadena productiva de la mora .

Tabla 2. Puntos críticos detectados en las buenas prácticas agrícolas.

\begin{tabular}{|c|c|c|c|}
\hline Puntos Críticos en las BPA & & Frecuencia & $\%$ \\
\hline \multirow{2}{*}{ Mejora Genética } & $\mathrm{Si}$ & 11 & 55 \\
\hline & No & 9 & 45 \\
\hline \multirow{4}{*}{ Tipo de Riego } & Goteo & 0 & 0 \\
\hline & Gravedad & 0 & 0 \\
\hline & Aspersión & 19 & 95 \\
\hline & Manual & 1 & 5 \\
\hline \multirow{3}{*}{ Influencia de lluvias } & Afectación por invierno & 4 & 20 \\
\hline & Es bueno & 3 & 15 \\
\hline & Hongos por exceso de lluvias & 9 & 45 \\
\hline \multirow{8}{*}{ Tipos de Poda } & De formación & 1 & 5 \\
\hline & De producción & 0 & 0 \\
\hline & De despunte & 1 & 5 \\
\hline & Utiliza todos los tipos de poda & 9 & 45 \\
\hline & De limpieza, formación y producción & 4 & 20 \\
\hline & De limpieza producción y despunte & 2 & 10 \\
\hline & De limpieza y despunte & 2 & 10 \\
\hline & De formación y producción & 1 & 5 \\
\hline \multirow{3}{*}{ Distancia de siembra } & $2 * 2$ & 13 & 65 \\
\hline & $3+3$ & 6 & 30 \\
\hline & $2 * 3$ & 1 & 5 \\
\hline \multirow{2}{*}{ Tipo de amarre } & Amarres & 5 & 25 \\
\hline & No realiza amarres & 15 & 75 \\
\hline \multirow{2}{*}{ Tutorado } & $\mathrm{Si}$ & 4 & 20 \\
\hline & No & 15 & 75 \\
\hline \multirow{2}{*}{ Desinfección de herramientas } & $\mathrm{Si}$ & 7 & 35 \\
\hline & No & 13 & 65 \\
\hline
\end{tabular}


Los resultados de la tabla 2 indican que el $85 \%$ de los agricultores no realiza análisis de agua, lo que es inadecuado según la normatividad del ICA (2016). Es recomendable hacer estudios sobre la cantidad de minerales que se encuentran en el agua, y estos estudios deben realizarse en periodos anuales, para evitar contaminar los cultivos con residuos químicos o biológicos (Ortiz-Villota, Romero-Morales \& Mesa-Rodríguez, 2018).

Actualmente, el 95\% de los productores realizan riego por aspersión para disminuir costos. Sin embargo, lo recomendado es el riego por goteo que evita la erosión del suelo y el ataque de hongos, mejorando a la vez la calidad y el rendimiento del producto (ICA, 2016). Otro aspecto es la afectación que causa el invierno, por el exceso de lluvia, debido a que hay mayor propagación de las enfermedades generadas por patógenos (Manzo-Sánchez et al., 2014). Sin embargo, esta afectación está relacionada con la deficiencia en los procesos de podas, amarres y tutorados al igual que con la falta de drenajes dentro del cultivo (Moreno-Medina, Casierra-Posada \& Blanke, 2016).
Es trascendental la desinfección de herramientas ya que solo el $35 \%$ de los agricultores las realizan en forma eficiente. Según los encuestados, las podas se realizan de forma diversa, pero no se hace de manera técnica y a los residuos de las podas no se les da la disposición adecuada. Para separar los cultivos, un $65 \%$ de los productores utilizan distancias de siembra de $2 \times 2 \mathrm{~m}$., cuando lo recomendable son distancias de 3 metros entre hileras y 2 entre plantas. Esto último mejora la aireación del cultivo y reduce el ataque de plagas, evitando el uso de pesticidas (Grijalba-Rativa et al., 2010).

En cuanto a la variable postcosecha, los indicadores demuestran puntos críticos en todos los aspectos investigados. No se cuenta con infraestructura adecuada para la recolección y empaque de la fruta, ni tampoco con los medios de transporte que se requieren. Adicionalmente, en el proceso de recolección no se usan recipientes ni bolsas de acuerdo a las normas técnicas. Además, la carencia de normas contables y administrativas impide tener información sobre la rentabilidad del cultivo, ver Tabla 3.

Tabla 3. Puntos críticos en la Postcosecha de la mora.

\begin{tabular}{llll}
\hline Postcosecha & & Frecuencia & $\%$ \\
\hline Recipientes de recolección & Cesta & 4 & 20 \\
& Canasta & 8 & 40 \\
& Recipiente plástico & 5 & 25 \\
& Cuñetes & 1 & 5 \\
Infraestructura Disponible & Canasta y cuñete & 1 & 5 \\
& Canastas y recipiente plástico & 1 & 5 \\
& Letrina & 3 & 15 \\
Bodega para empaque & Casetillas & 1 & 5 \\
Manejo de Empaque & Anaqueles & 2 & 10 \\
& Bodega & 2 & 10 \\
& Ninguna & 12 & 60 \\
& Si & 3 & 15 \\
& No & 17 & 85 \\
& Canastillas & 4 & 20 \\
& No manejan empaques & 4 & 20
\end{tabular}




\begin{tabular}{|c|c|c|c|}
\hline \multirow[t]{6}{*}{ Transporte } & Vehículo abierto & 6 & 30 \\
\hline & Vehículo cerrado & 2 & 10 \\
\hline & Vehículo abierto y caballo & 2 & 10 \\
\hline & Caballo & 6 & 30 \\
\hline & Manual & 2 & 10 \\
\hline & No responde & 2 & 10 \\
\hline \multirow[t]{5}{*}{ Higiene del productor } & Lavado contante de manos y cambio de ropa diario & 2 & 10 \\
\hline & Baño por la mañana y lavado constante de manos & 5 & 25 \\
\hline & Lavado constante de manos & 1 & 5 \\
\hline & $\begin{array}{l}\text { Baño por la mañana, lavado contante de manos y cam } \\
\text { bio de ropa diario }\end{array}$ & -11 & 55 \\
\hline & Fuma & 1 & 5 \\
\hline \multirow[t]{5}{*}{ Registros } & Técnicos & 2 & 10 \\
\hline & Técnicos y gastos e ingresos & 1 & 5 \\
\hline & Producción, gastos e ingresos & 1 & 5 \\
\hline & No llevan & 12 & 60 \\
\hline & Técnicos, producción, gastos e ingresos. & 4 & 20 \\
\hline \multirow{4}{*}{$\begin{array}{l}\text { Problemas en los procesos de } \\
\text { producción/comercialización }\end{array}$} & Falta de recursos & 1 & 5 \\
\hline & Intermediarios & 6 & 30 \\
\hline & Falta de tecnificación & 4 & 20 \\
\hline & No responde/Sin comercialización & 9 & 45 \\
\hline
\end{tabular}

La entrevista a expertos se analizó en 3 categorías: características de la cadena productiva; fortalezas y oportunidades; y debilidades del sector. En cuanto a la investigación agrícola, se encontró que no se han buscado alternativas que permitan mejorar genética, nutricional y medicinalmente los cultivos. Los expertos sugieren fortalecer las ventajas nutricionales de la mora, para darle valor agregado y para impulsar su transformación industrial, ya sea en presentaciones tradicionales - p.ej. mermeladas, pulpas y jugos-, o en innovaciones para el sector de alimentos. Para lograrlo, se puede aprovechar la existencia de espacios para aumentar la cantidad de siembra del cultivo y fortalecer las ventajas del producto, con la aplicación de caldos nutricionales orgánicos.
Respecto a las BPA, los expertos entrevistados no coinciden con los productores en que los cultivos se realicen cumpliendo los estándares de agricultura verde u orgánica. Aunque se hacen esfuerzos por lograrlo, no se están llevando a cabo con los requerimientos técnicos establecidos para que sea un producto totalmente orgánico. En cuanto a los puntos críticos de las prácticas agrícolas, productores y expertos son conscientes de las dificultades existentes y de la falta de planes de mejoramiento para ejecutar en el corto o mediano plazo. Sobre el tema de postcosecha, existe acuerdo en que el procedimiento de recolección, selección y empaque se realiza en forma tradicional y no cumple con las normas técnicas. Asimismo, hay concenso en que la existencia de intermediarios disminuye las ganancias (Ver Figura 2). 


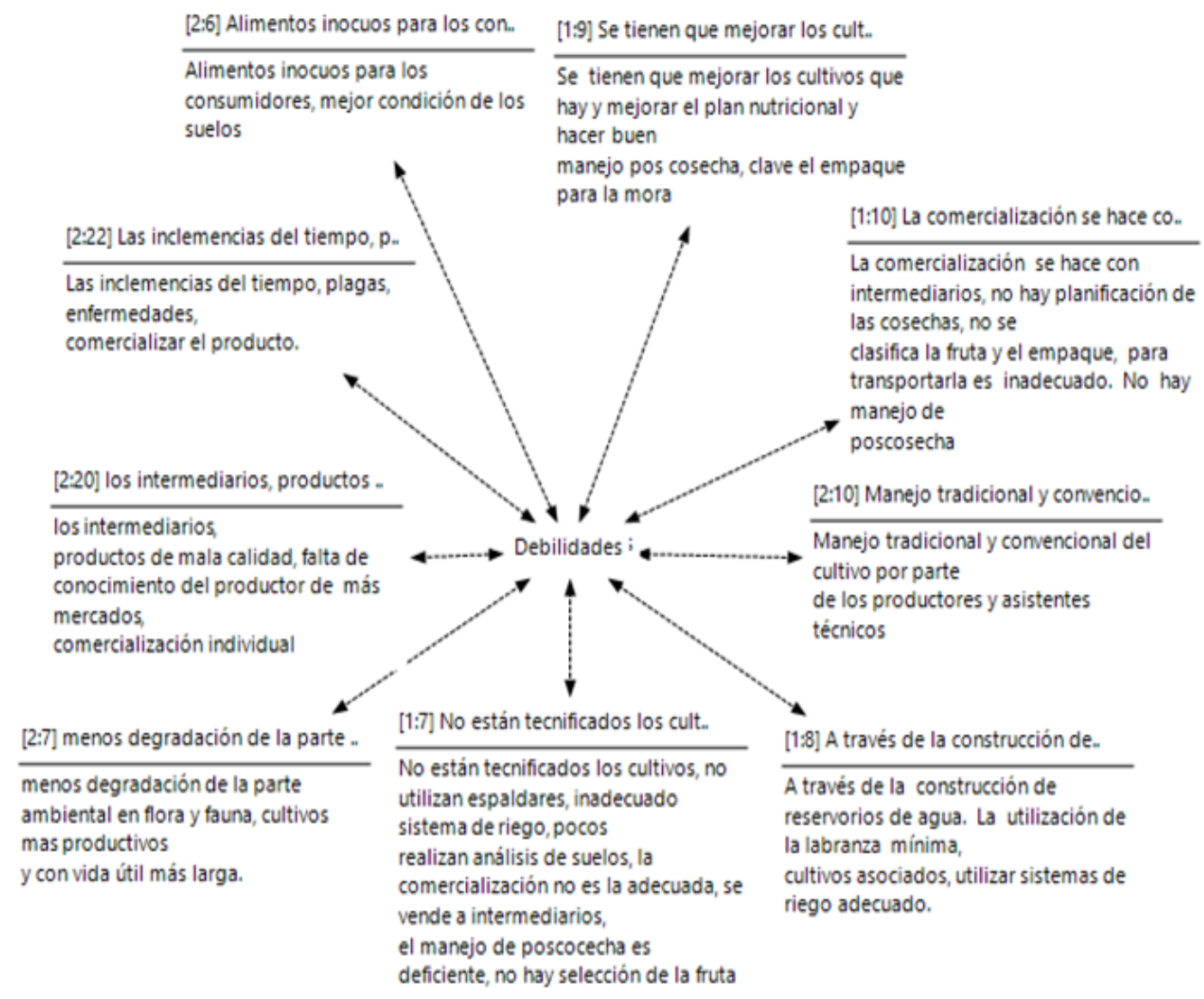

Figura 2. Debilidades en la producción de la mora según los expertos.

En vista de lo anterior, para fortalecer los puntos críticos que existen actualmente en la cadena productiva de la mora, se requieren cambios en los procesos de productividad. Entre ellos se tienen: fortalecer la asociatividad, cualificarse en los procesos de comercialización, así como fortalecer el producto desde la genética y la producción orgánica (Ver Figura 3). 


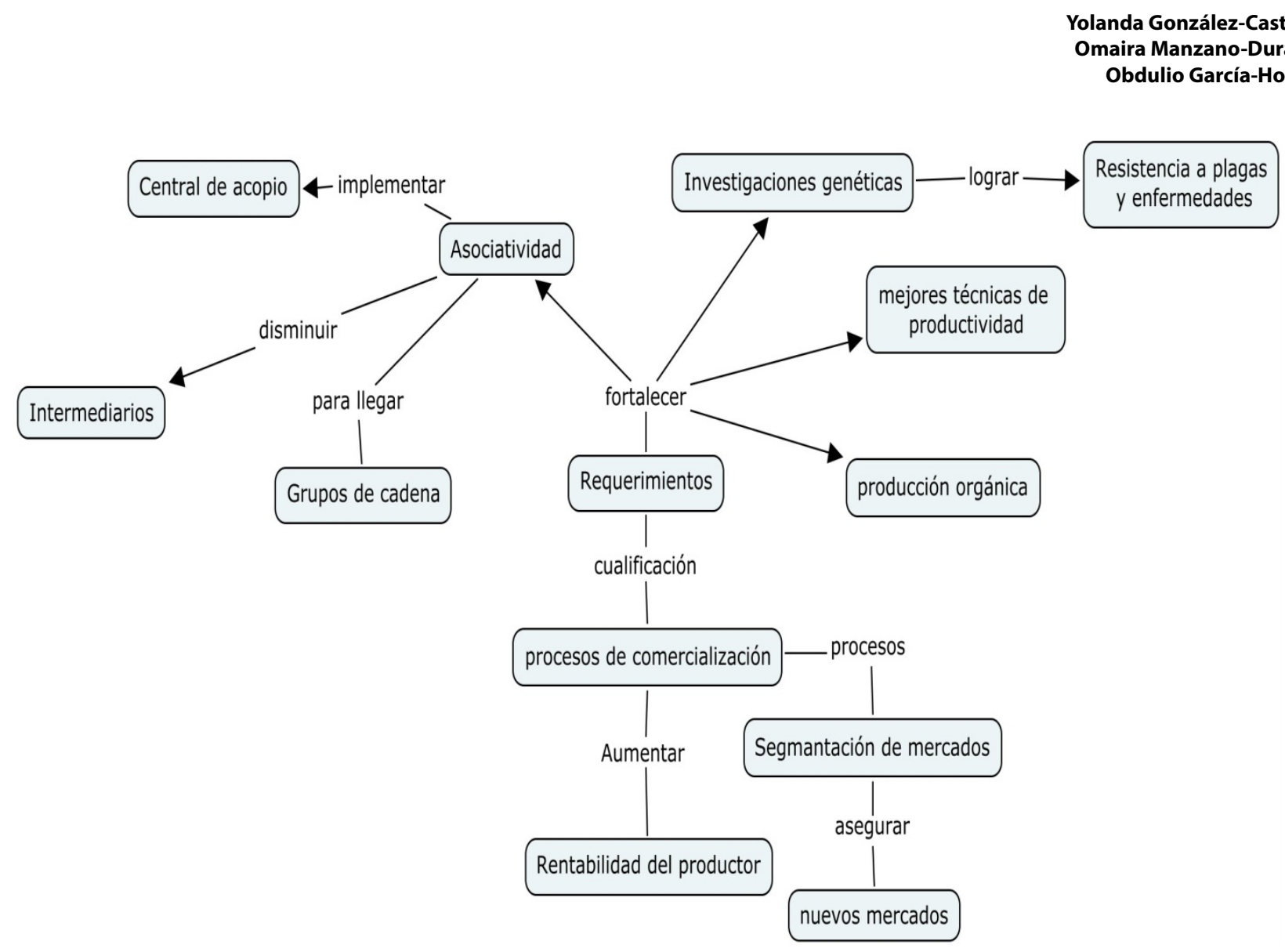

Figura 3. Requerimientos para la producción de la mora según los expertos.

En cuanto a la asociación, se requiere aumentar el número de socios para tener suficiente cantidad de producto al momento de comercializar y crear alianzas estratégicas, que les permita realizar ventas a gran escala. También es importante diversificar la producción para atender diferentes segmentos de mercado e impulsar la venta directa, eliminando el intermediario, como lo indica la investigación de López-Posada y Pachón-Ariza (2017). El aumento de la productividad y la competitividad requiere mejoras en la calidad del producto con la tecnificación, y crear reservorios y drenaje para el manejo adecuado del agua, evitando su desperdicio.

\subsection{Discusión}

Los resultados anteriores coinciden con los hallazgos de Bonilla y Parada (2018), quienes en un estudio de mercado de la cadena productiva de la mora en Pamplona, encontraron: baja rentabilidad, comercialización deficiente y el no poseer los medios necesarios para tecnificar la producción. Esto último se debe a problemas en los cultivos, como: ataque de plagas, no se podan ni se tutoran, les falta tecnificación y riego, altos costos de producción, dependencia de las épocas de lluvia, precios inestables, dificultad para comercializar y explotación marginal (Corpoica, 2014). Igualmente, el sector requiere: diversificación de la variedad, mejoras en la calidad genética del material de siembra, control de las condiciones fitosanitarias, aumentar el valor nutricional y medicinal de la fruta (Grijalba-Rativa et al., 2010). 
En cuanto al mejoramiento genético, Rodríguez-Barona, Giraldo y Zuluaga (2015), afirman que al adicionar microorganismos probióticos con fibra en matrices vegetales como la mora Castilla, mediante técnicas como impregnación al vacío o conservadas por liofilización, se obtiene un alimento con cualidades simbióticas y a su vez favorece la preservación del producto. Igualmente, a partir de los cultivos in vitro y de la creación de bancos de germoplasma, es posible aumentar la productividad y ofrecer materiales de diferentes orígenes y con diferentes características a los cultivadores de Mora (Agencia Iberoamericana para la difusión de la ciencia y la tecnología, 2011).

Adicionalmente, dada la importancia del cuidado del ambiente, es necesario implementar técnicas de producción agrícola direccionadas al uso eficiente de los recursos, que permitan una agricultura sostenible. En este sentido, la aplicación de abonos orgánicos es una gran oportunidad para los agricultores (Albarracín-Sánchez et al., 2018). La agricultura orgánica se caracteriza por la no presencia de fertilizantes sintéticos y pesticidas, así como por el empleo de materia orgánica para mantener la fertilidad de la tierra (Bettiol, Ghini, Haddad \& Siloto, 2004). Ejemplo de ello es un abono foliar orgánico en forma líquida, denominado biofertilizante, que resulta de un proceso de fermentación anaeróbica de restos orgánicos de animales y vegetales, el cual contiene nutrientes que fortalecen el crecimiento, desarrollo y producción en las plantas (Toalombo, 2013). Sin embargo, el desconocimiento por parte de los agricultores de la mora de castilla acerca de los beneficios de este tipo de biofertilizantes, impide su aplicación en los cultivos.

En cuanto a las BPA, se presentan algunas recomendaciones para los productores, en aquellos factores en los que se encuentran más débiles. El cultivador requiere conocer el tipo de fuente de agua que está utilizando y los riesgos potenciales que esta puede acarrear, tanto para la planta como para la salud humana. Según la Organización de las Naciones Unidas para la Alimentación y la Agricultura, FAO (2014), se debe, en lo posible: utilizar filtros y documentar los resultados del análisis del agua, así como proteger los cauces de agua. Los métodos de riego más recomendados para el cultivo de mora, son: el goteo, micro aspersión a baja altura e inundación (Gobernación de Antioquia, 2014).

Otra práctica para el cultivo de la mora, poco utilizada, es el tutorado. Este consiste en guiar a la planta para que no se mantenga en el suelo sino que se eleve y de esta forma pueda tomar el aire y mejorar el cultivo (Pérez, 2011). Igualmente importante en el cultivo de la mora es la poda, que favorece el proceso reproductivo (floración y formación de frutos), al igual que favorece la renovación del aire (Moreno-Medina, Casierra-Posada \& Blanke, 2016).

Las BPA también se relacionan con la administración de las fincas o terrenos productivos. En tal sentido, es importante llevar registros de campo, con su ubicación, nombre del representante legal, razón social y datos de contacto. Así mismo, es necesario: registrar los cultivos con sus fechas de siembra, existencia de parásitos o de otras enfermedades, manejo de productos sanitarios, higiene del predio y del personal. Igualmente, se requiere información sobre las cantidades de siembra y cosecha, así como llevar registros contables de todo el proceso productivo, para que los agricultores puedan tomar decisiones adecuadas al momento de la venta (FAO, 2014).

Lo anterior permitiría mantener una producción permanente y de calidad, ampliar la producción con nuevos cultivos, utilizando así buenas prácticas agrícolas que faciliten la aplicación de la agroindustria para la transformación del producto. Esto, a su vez, permitiría encontrar soluciones a los puntos críticos de la cadena productiva de la mora (Rubus glaucus Benth), en el municipio de Pamplona. 


\section{Conclusiones}

La investigación permitió encontrar los puntos críticos de la cadena productiva de la mora (Rubus glaucus Benth), en el municipio de Pamplona, tales como: realizar y aplicar estudios para la mejora genética de los cultivos, así como implementar buenas prácticas para el análisis de agua y desinfección adecuada. Igualmente, se requiere un manejo técnico en cuanto al riego, distancias de siembra, uso de aporques, amarres y tutorados, estandarización de implementos para la recolección y transporte del producto, junto con el uso de prácticas administrativas y contables.

Los resultados brindan información relevante en cuanto a: investigación, buenas prácticas agrícolas y manejo de poscosecha, para tomar medidas que permitan fortalecer la competitividad del cultivo de la mora. En este sentido, es necesario dinamizar la cadena productiva de la mora, impactando así en el desarrollo económico y social de la región $y$, especialmente, en la calidad de vida de los productores.

Se requieren nuevas investigaciones en el área, tales como una vigilancia estratégica e inteligencia competitiva y para el producto, con apoyo de entes gubernamentales. Esto permitirá determinar los avances de otras regiones, estableciendo estrategias puntuales para el municipio de Pamplona. Además, es importante realizar estudios bioquímicos del producto, para conocer sus diferencias competitivas y potenciarlas hacia el mercado.

\section{Agradecimientos}

Este trabajo es un avance del macroproyecto: inteligencia competitiva de la cadena productiva de la mora (Rubus glaucus Benth), del grupo de investigación GRINDES, apoyado por la Maestría en Administración de Organizaciones, de la UNAD.

\section{Referencias}

Agencia Iberoamericana para la Difusión de la Ciencia y la Tecnología (2011). Agricultores y Científicos unieron esfuerzos para optimizar el cultivo de uno de los frutos más tradicionales de Colombia. Recuperado de: http://www.dicyt.com/viewNews. php?newsld=27367

Albarracín-Sánchez, D. M., Roa-Parra, A. L., Solano-Ortega, F., \& Montañez-Acevedo, G. (2018). Producción de abono orgánico mediante el compostaje aerotérmico de residuos de poda. Bistua, 16 (1), 156-162. Recuperado de: http://revistas. unipamplona.edu.co/ojs_viceinves/index.php/ BISTUA/article/view/3203

Asohofrucol (2008). Los Santanderes y Cesar en el Plan Frutícola nacional. Frutas y Hortalizas, 1 (6), 14-23.

Ayala, L., Valenzuela, C., \& Bohórquez, Y. (2013). Variables determinantes de la madurez comercial en la mora de Castilla (Rubus glaucus BENTH). Scientia Agroalimentaria, 1, 39-44. Recuperado de: http://revistas.ut.edu.co/index.php/scientiaagro/ article/view/29/0

Bettiol, W., Ghini., R., Galvão, J., \& Siloto, R. (2004). Organic and conventional tomato cropping systems. Scientia Agricola, 61 (3), 253-259. Recuperado de: https://dx.doi.org/10.1590/S010390162004000300002

Bonilla, L., \& Parada, R. (2018). Plan estratégico de mercado para la cadena productiva de la mora (Rubus glaucus Benth). Revista distancia al día, 8, 6-17. Recuperado de: https://repository.unad.edu. co/bitstream/10596/20837/3/1094264614.pdf

Corporación Colombiana de Investigación Agropecuaria, Corpoica (2014). Principales característi- 
cas y tendencias del mercado de cítricos en Colombia. Bogotá, Colombia.

Dotor-Robayo, M. Y., González-Mendoza, L. A., Castro, M. A., Morillo-Coronado, A. C., \& Morillo-Coronado, Y. (2016). Análisis de la diversidad genética de la mora (rubus spp.) en el departamento de boyacá. Biotecnología en el Sector Agropecuario y Agroindustrial, 14 (2), 10-17. doi: http://dx.doi. org/10.18684/BSAA(14)10-17

Gobernación de Antióquia (2014). Manual técnico del cultivo de la mora bajo buenas prácticas agrícolas. Medellín, Colombia.

Gobernación Norte de Santander (2016). Plan de desarrollo para Norte de Santander 2016-2019. Cúcuta, Colombia.

Grijalba-Rativa, C. M., Calderón-Medellín, L. A., \& Pérez-Trujillo, M. M. (2010). Rendimiento y Calidad de la Fruta en Mora de Castilla (Rubus glaucus Benth), con y sin Espinas, Cultivada en Campo Abierto en Cajicá (Cundinamarca, Colombia). Revista Facultad De Ciencias Básicas, 6 (1), 24-41. doi: https://doi. org/10.18359/rfcb.2079

Instituto Colombiano Agropecuario, ICA (2016). Resolución N020009 para la certificación de buenas prácticas agrícolas en producción primaria de vegetales y otras especies para consumo humano. Bogotá, Colombia. Recuperado de: https://www. ica.gov.co/Normatividad/Normas-Ica/Resoluciones-Oficinas-Nacionales/RESOLUCIONES-DEROGADAS/Res-020009-DE-2016.aspx

Kessel-Domini, A. (2012). Mejora genética de la fresa (Fragaria ananassa Duch.), a través de métodos biotecnológicos. Cultivos Tropicales, 33 (3), 34-41. Recuperado de: http://scielo.sld.cu/scielo.php?scrip$\mathrm{t}=$ sci_abstract\&pid=S0258-59362012000300005

Llerena, W., Samaniego, I., Ramos, M., \& Brito, B. (2014). Caracterización fisicoquímica y funcional de seis frutas tropicales y andinas ecuatorianas.
Alimentos, Ciencia e Ingeniería, 22 (2), 13-22. Recuperado de: http://repositorio.iniap.gob.ec/handle/41000/3279

López-Posada, J. C., \& Pachón-Ariza, F. A. (2017). Identificación de ventajas y desventajas de los canales de comercialización en las economías campesinas de dos municipios de Meta y Cundinamarca, Colombia. Revista de Investigación, Desarrollo e Innovación, 8 (1), 35-47. doi: 10.19053/20278306. v8.n1.2017.7369

Manzo-Sánchez, G., Orozco-Santos, M., Martínez-Bolaños, L., Garrido-Ramírez, E., \& Canto-Canche, B. (2014). Enfermedades de importancia cuarentenaria y económica del cultivo de banano ( Musa sp.) en México. Revista mexicana de fitopatología, 32 (2), 89-107. Recuperado de: http://www. scielo.org.mx/scielo.php?script=sci_arttext\&pi$\mathrm{d}=$ S0185-33092014000200089\&lng=es\&tlng=es.

Ministerio de Agricultura y Ganadería de Costa Rica (2014). Mora (Rubus spp) cultivo y manejo poscosecha. Recuperado de: http://www.mag.go.cr/ bibliotecavirtual/F01-8862.pdf

Miranda-Lasprilla, D. (2011). Estado actual de fruticultura colombiana y perspectivas para su desarrollo. Revista Brasileira de Fruticultura, 33 (spe1), 199-205. doi: https://dx.doi.org/10.1590/S010029452011000500023

Moreno - Medina, B., Casierra-Posada, F., \& Blanke, M. (2016). Índices de crecimiento en plantas de mora (Rubus alpinus Macfad) bajo diferentes sistemas de poda. Revista Colombiana de Ciencias Hortícolas, 10 (1),28-39. doi: https://doi.org/10.17584/ rcch.2016v10i1.4457

Organización de las Naciones Unidas para la Alimentación y la Agricultura, FAO. (2014). Agricultura familiar en América latina y el Caribe: Recomendaciones de política. Santiago de Chile, Chile. Recuperado de: http://www.fao.org/docrep/019/i3788s/ i3788s.pdf 
Ortiz-Villota, M. T., Romero-Morales, M. A., \& Meza-Rodríguez, L. D. (2018). La biorremediación con microalgas (Spirulina máxima, Spirulina plantensis y Chlorella vulgaris) como alternativa para tratar la eutrofización de la laguna de Ubaque, Colombia. Revista de Investigación, Desarrollo e Innovación, 9 (1), 163176. doi: https://doi.org/10.19053/20278306. v9.n1.2018.8153

Pérez, V. (2011). Plan de fertirrigación en el cultivo de mora de castilla con espinas (Rubus glaucus $B$ ), Cantón Ambato, provincia de Tungurahua (Tesis de grado). Ambato, Ecuador: Universidad Técnica de Ambato. Recuperado de: http://repositorio.uta. edu.ec/jspui/handle/123456789/881

Rodríguez-Barona, S., Giraldo, G., \& Zuluaga, Y. (2015). Evaluación de la Incorporación de Fibra Prebiótica sobre la Viabilidad de LactobaciHus casei Impregnado en Matrices de Mora (Rubus glaucus). Información tecnológica, 26 (5), 25-34. doi: http:// dx.doi.org/10.4067/S0718-07642015000500005

Sánchez-Morales, J. A., Villares-Jibaja, M. X., Niño-Ruiz, Z., \& Ruilova, M. B. (2018). Efecto del piso altitudinal sobre la calidad de la mora ( Rubus glaucus benth) en la región interandina del Ecuador. Idesia (Arica), 36 (2), 209-215. doi: https://dx. doi.org/10.4067/S0718-34292018005000702

Toalombo, M. (2013). Aplicación de abonos orgánicos líquidos tipo biol al cultivo de mora (Rubus glaucus Benth) (Tesis de grado). Ambato, Ecuador: Universidad Técnica de Ambato. Recuperado de: http://redi.uta.edu.ec/handle/123456789/6490

Trujillo-Navarro, Y. (2018). Conservación de la fresa (fragaria ananassa) variedad camarosa a partir de la modificación pasiva de la atmósfera. Infometric@ - Serie Ingeniería, Básicas y Agrícolas, 1 (2). Recuperado de: http://www.infometrica.org/index. $\mathrm{php} / \mathrm{syh} /$ article/view/29

Usca, J (2011). Evaluación del potencial nutritivo de mermelada elaborado a base de remolacha. Recuperado de: http://dspace.espoch.edu.ec/bitstream/123456789/1165/1/56T00265.pdf

Zuluaga-Duque, J. F. (2017). Relación entre conocimientos, saberes y valores: un afán por legitimar los saberes más allá de las ciencias. Revista de Investigación, Desarrollo e Innovación, 8 (1), 61-76. doi: 10.19053/20278306.v8.n1.2017.5973 
